\title{
Using Feature Extraction and Classification Methods of Movie Opinions Predication
}

\author{
S Nirupama, Pamireddy Sindhu, N. Divya Sri, P. Lakshmi Durga Bhavani
}

\begin{abstract}
Film rankings and analysis at sites like IMDb (Internet Movie Database) square measure ordinarily employed by picture show goers to make your mind up that movie to look at or obtain next. Currently, picture show goers base their choices on that movie to look at by staring at the ratings of films in addition as reading a number of the reviews at IMDB. Sentiment analysis could be a different field of different opinion where the methods of analysis are targeted on feature extraction and selection technique of emotions and opinions of the individual's audience towards selected methods from semi-structured, structured or unstructured matter information. This paper, we focus on our techniques of sentimental analysis on IMDB picture show review information. To survey the sentimental words method to classify the polarity of the picture show review on a scale of highly dislikes highly liking and performing different extraction feature and positioning of reviews. It uses these options to train our multilable classifier to classify the picture show review into its correctable.
\end{abstract}

Keywords: Feature Extraction and Selection, Opinion Mining, Sentiment Analysis, Movie Review.

\section{INTRODUCTION}

Information has been developing at companion in nursing exponential price giving upward jostle to a substitute alternate to full of it inside which customer's particular their opinion throughout channels like facebook, twitter and other social web sites. Movie goers or customers or reviewers utilize film scores and critiques in selecting their next film to see/watch. Opinions that rectangular measure being expressed inside the kind of evaluations provide a threat for manufacturer spanking new analysis to seek out aggregate abhorrences and preferences of online networking assets. The area of film evaluations is one such area of critiques that affects everybody from audience movie critics to Assembly Community. The critiques of the movie being denote on web sites are not formal opinions then again rectangular measures rather terribly casual and rectangular measure unstructured sort of synchronic linguistics. Within the next few years

Revised Manuscript Received on December 30, 2019.

* Correspondence Author

Nirupama S, Assistant Professor, Dept. of IT, SNIST, Hyderabad, Telangana, India. Email:nirupamas@sreenidhi.edu.in

Pamireddy Sindhu, B. Tech Graduate Student, Dept. of IT, SNIST, Hyderabad, Telangana, India. sindhupamireddy@gmail.com

N. Divya Sr, B. Tech Graduate Student, Dept. of IT, SNIST, Hyderabad, Telangana, India. nethidivyasri@gmail.com

P. Lakshmi Durga Bhavani, B. Tech Graduate Student, Dept. of IT, SNIST,Hyderabad,Telangana, India. lakshmidurga0617@gmail.com

(C) The Authors. Published by Blue Eyes Intelligence Engineering and Sciences Publication (BEIESP). This is an open access article under the CC BY-NC-ND license (http://creativecommons.org/licenses/by-nc-nd/4.0/) sentiment analysis may want to be a science which will be indispensable. We are in a situation to recognize awful substance from top notch information with feeling AI. In view of the procedures we can perceive if a motion picture has additional great assessment than unfortunate supposition and perceive the clarifications why these sentiments are phenomenal or awful.

In our exploratory calculation used to movie investigation from IMDb the world's most predominant material hotspot for film and superstar objects [1] with overviews for more than 3.5 million movies.In IMDb people give reviews and qualities scores for different reviews. In any case the all things considered rating of a movie is determined by IMDbs guarantee rating computation which thus depends on examiner scores [2].

A report might also additionally comprise a combination of wonderful and bad opinions. Sensibility diploma audience opinion mining is performed through two duties i.e. Objective and Subjective.

Objective: I pick up a new smart watch few years ago.

Subjective: Its good watch. The polarity of the opinion is determined by using the subjective sentence.

Positive: Nice mobile.

Negative: Has poor radio signal reception quality.

In this paper we convey with a Convolutional Neural Networks (CNN) using to determine the well-known polarity of film review. To analyze and learn about the factors that has an impact on the sentiment rating of the film contrast text. In state-of-the-art years sentiment evaluation functions have opened out to almost each potential domain. Wistful evaluation grouping assumptions in printed content into preparing predominantly rely upon notion extremity. Duality potential the realm of having two opposite estimations assessments or perspectives. In this polarity can be positive terrible or unbiased there is moreover a four kind a effective in which sentiment holders supply guidelines to enhance that aspect opinion estimate aims to decide the attitude of a speaker or a creator with regard to a few subject or the widespread relevant extremity of a report. The attitude might moreover be their judgment or valuation full of feeling state which is the passionate country while composing or the expecting passionate communication which is the enthusiastic impact the author should have on the per user. Sentiment is depicted as any assortment of feeling and within the setting of assumption assessment is the conclusion that being communicated within the structure of content or discourse. Feature resolution: most analyzers examine widespread characteristic selection in their strategy to enhance overall estimation act with few using more advanced ways. only few papers are entirely focusing to beautify the characteristic resolution of sentiment analysis.

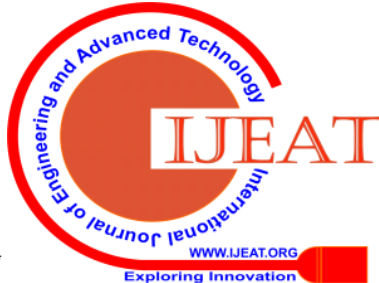


Pang lee [7] is one among them who eradicated sentences on a test bed consisting of impersonal and personal textual content trained on system. AT the establishing they seen that quit end result of sentiment classification has been clearly decreased,

Then they concluded it was extra in all likelihood that sentences adjacent to discontinued sentences extended classification quit result over their control. Another work used state-of-the-art attribute selection and located that the use of all information acquire and genetic method concludes in an enchantment efficiently.

Convolutional Neural Network (CNN): In work area learning a CNN compose might be a kind of feed ahead phony neural mastermind the put the individual neurons are tiled so that they respond to covering areas inside the visual field. These systems have been impelled with the assistance of natural techniques and are assortments of multilayer approach sketched out to utilize irrelevant measures of preprocessing [3]. Consenting to Yoon Kim [4] he had performed tests with CNN structures dependent on beat of word2vec. Despite small tuning of hyper parameters a direct CNN with one layer of convolution performs incredibly well. He besides incorporates to the settled affirmation that solo pretraining of word vectors is a fundamental fixing in significant learning for NLP.

This segment describes about the issue of opinion mining of movie reviews:

Gathering Emotion Words - it is the estimation; each one of the evaluation explanation comprises which have a fundamental contribution in duality of the review. For example the interesting right here the opinion words explains us about the duality of the film is positive.

Contempt- documentary statements are really hard to known surely it cannot be said bad or well. For ex: this movie was bad.

Parsing - What does the participle and/or non-objective or unbiased documentary statements indeed belong to?

Comparing - What capacity of data in the entire world of clients is entered?10\% offers you a hard concept of what's going on nowhere close to the decision you get critiques.

\section{RELATED WORK}

Godbole manjunath stevens [5] current a machine that enables wonderful or poor assumption to every wonderful item in the text collection. there are two phases in their machine a sentiment awareness section the place where idea expressing substance are decided and a scoring segment where a corresponding rating for each item is resolved. web network sources like imdb, twitter [6] and facebook are currently being engaged by the vast majority of the supposition assessment inquire about on requiring the strategies be customized to introduce the developing intrigue of judgment in the state of content, besides accomplishing the expression level assessment of film audits demonstrates to be a difficuit work.

Function specific sentiment analysis is used to inspect the product overview by S. Mukherjee and P. Bhattacharyya et.al. [8] to size of relation between the facets and related opinions they used dependency parsing approach.a system was developed by them which extracts the conceivable points from evaluate and batch assessment explanation defining all of the factors .Two datasets lakka raju et. al. [9] and hu and liu et. al. [10] were used for achieving experimental outcome. The considered machine fulfills common veracity of $80.98 \%$ for dataset 1 and $70 \%$ for dataset.

Haruna isah and paul trundle et al. [11] proposed a lexicon primarily based and laptop gaining knowledge of primarily based sentiment classification approach which makes use of naïve bayes most entropy and assist vector computing device desktop getting to know analyzers. A framework was developed by them to measures the experiences of consumer of well-known brands of drugs and cosmetics. For attaining other analyzers naive bayes classifiers are used to attain basic end outcome.

\section{PROPOSED METHODOLOGY}

Data preparation: gathering of critiques of any unique product from different sources like internet or any online sites is known as data preparation. The accumulated information might also be either disorganized. Sentiment analysis procedure can utilize any publically convenient datasets which outfit the huge assortments of sentiments. The subset of assessments contains disconnected information at some point, for instance: html labels and url records and so on at the phase of preprocessing of audits expulsion of such futile records is practiced.

Preprocessing: Its use to put off clattering inconsistent and unfinished records. For the formation of textual content preprocessing and characteristic extraction is as important phase. It reduces and removes the noisy, inconsistent and unfinished data. In appropriate statistics eradicated and after getting these applicable statistics we can pick out and extract aspects from that and that aspects will advocated for the classification. In Figure.1 preprocessing is clearly described.

Stemming: It is the process of removing prefixes to affixes word, this is called stem root. For ex: develop, develops, and developed these words are stem root. Porter stemming algorithm is used in this method.[12]

$$
\begin{aligned}
& {[C](V C) m[V]} \\
& \text { It denotes: } \quad C \rightarrow \text { Optional Contents } \\
& (V C) \rightarrow \text { Vowel (V) followed by a consonant } \\
& \text { (C) m times. }
\end{aligned}
$$

Stopping: This is the technique which removes the most common word in sentence. And it also reduces the size of document. 

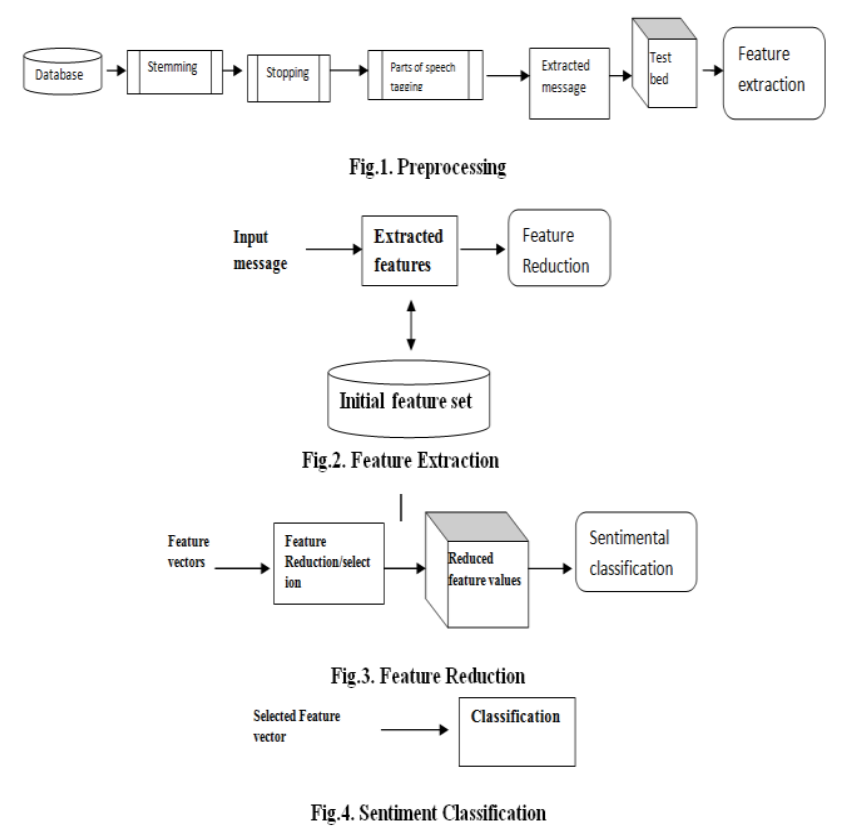

Review analysis: This progression assesses the unprecedented components of sentiments and afterward recognizes the captivating insights in the diagram together with supposition. Audit assessment step initially applies an assortment of calculation obligations to the assessment and afterward separates the feeling and purposes of the item. Two customarily utilized systems in assess assessment are POS labeling and refutation labeling.

Feature Selection and Extraction Feature Selection: The procedure of picking exclusively relevant viewpoints to usage in mannequin advancement. Highlight extraction potential when the records given to a calculation is too overwhelming to even think about handling and it is suspected to be immaterial then it tends to be changed over into a diminished arrangement of highlights. In Fig.2 Feature determination is depicted.

Sentiment Polarity: Its capacity is to classify the sentiment evaluations as nice or bad reviews

Sentiment Classification: Two essential techniques use for classifying overview are sentiment orientation method and desktop studying method. Sentiment orientation consists of two btasks. First mission is to collect the opinions from different review. Second mission is to determine the usual sentiment orientation of a sentence. The sentiment orientation contains two seed adjective excellent and poor.

The laptop mastering method depends on the classification lessons such as +ve and -ve. Any classification algorithm is use to train and take a look at the model on the other hand for convenient sentiment analysis Naive Bayes (NB) help vector computer SVM and maximum entropy me algorithms are drastically used. In Fig.4 Sentimental classification is described.

Facts gathering in this records collects information are gathered from one-of-a-kind relevant sources such as blogs web crawling twitter tweets online evaluate newsfeeds record scanning etc. In quick have to replicate on consideration on sentimental file as per the diploma of sentiment analysis.

Proposed Algorithm: The algorithm is following as per the steps as mention below.
Step 1: Initialize all feature and reviews to 0.

Step2: Each Sentence is extracted from stemming and stopping methods.

Step 3: For each extract feature done

\section{If sentiment word are Negative, then}

We calculate no. of words to be negative sentiment word. Else we calculate no. of words to be positive sentiment word. Step 4: Determine labeling of No. of positive word and No. of negative word.

Step 5: Finally it evaluated and classify the sentiment reviews based on Classification techniques. This is final stage which we carried out in our proposed system.

\section{RESULT ANALYSIS}

We have collected 50000 reviews from IMDB data set, and this dataset have $50 \mathrm{~K}$ movie reviews. Dataset consists of all positive and negative reviews. The table.1. have complete IMDB dataset.

\begin{tabular}{|l|l|}
\hline Review & Sentiment \\
\hline $\begin{array}{l}\text { 49582 Unique values taken from IMDB } \\
\text { data set }\end{array}$ & $\begin{array}{l}\text { Positive } \\
\text { reviews 50\% }\end{array}$ \\
\cline { 2 - 2 } & $\begin{array}{l}\text { Negative } \\
\text { reviews 50\% }\end{array}$ \\
\hline
\end{tabular}

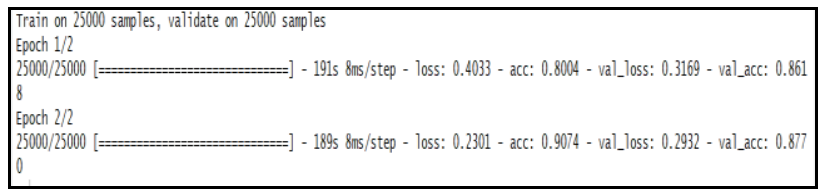

The comparison and classification analysis method used a naïve bayes. We randomly choose 25000 using this data set we are analyzing and classifying the review of audience. We got exact accuracy on these dataset.

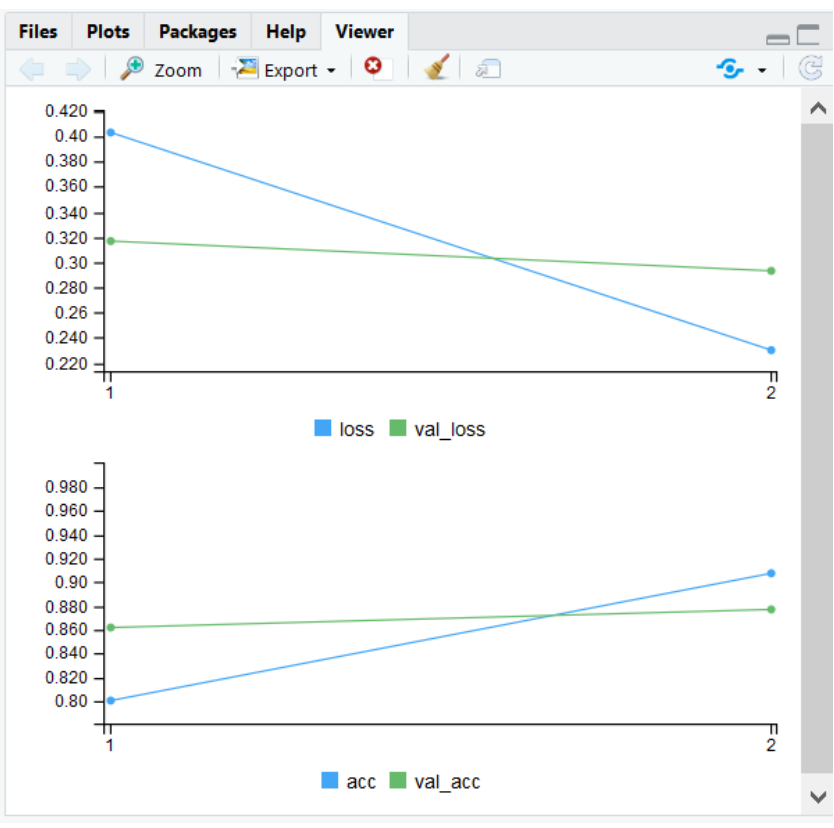

Published By:

Blue Eyes Intelligence Engineering \& Sciences Publication 


\section{CONCLUSION}

Sentiment analysis performs necessary position during the time making a choice toward a particular product is stated in this paper. But certain first-class measures like helpfulness and usefulness are very important to think about while inspecting each review. It can be utilized for any purposes that follow information mining regulations. despite the strategies and methods used for opinion evaluation are achieving fast and offering huge exceptional results lot of troubles in this self-discipline of analyze about stay unresolved and additionally it is challenging to detect the faux judgment with the aid of reading. fake critiques additionally considered as top notch exquisite consider now and again and it was once converted like no one can discover their original motive such as fake evaluation exposure is some other essential area which requires deep statistics mining techniques.

\section{REFERENCES}

1. IMDb, "What is IMDb." [Online]. Available: http://www.IMDB.com/pressroom/

2. IMDb, "The vote average for film." [Online]. Available: http://www.IMDb.com/help/show leaf? Votes

3. Https://en.wikipedia.org/wiki/Convolutional_neural_network

4. Kim, Yoon. "Convolutional neural networks for sentence classification." arxiv preprint arxiv:1408.5882 (2014).

5. Godbole, Namrata, manjasrinivasaiah, and Steven Skiena. "Large-Scale Sentiment Analysis for News and Blogs." ICWSM 7 (2007): 21.

6. Pak, Alexander, and Patrick Paroubek. "Twitter as a Corpus for Sentiment Analysis and Opinion Mining." LREC. Vol. 10. 2010

7. Pang, Bo, Lillian Lee, and shivakumar vaithyanathan. "Thumbs up?: sentiment classification using machine learning techniques." Proceedings of the ACL-02 conference on Empirical methods in natural language processing-Volume 10 . Association for Computational Linguistics, 2002.

8. Subhabrata Mukherjee, Pushpak Bhattacharyya," feature specific Sentiment Analysis for product Reviews", IET, 2015, IIT Bombay.

9. Himabindu Lakkaraju, Chiranjib Bhattacharyya, Indrajit Bhattacharyya and Srujana Merugu, "Exploiting Coherence for the simultaneous discovery of latent facts and associated sentiments", siam international Conference on Data Mining (SDM), April2011.

10. Minqing $\mathrm{Hu}$ and Bing Liu, "Miming and Summarizing customer reviews", KDD 04: proceedings of the tenth ACM SIGKDD international Conference on knowledge discovery and data mining.

11. Haruna isah, Paul Trundle, Daneiel Neagu, "Social Media Analysis for Product Safety and using Text Mining and SA", IET,2015,University of Bradford, UK

12. M.F. Porter, "An algorithm for suffix stripping", Program, vol. 14, 1980.

\section{AUTHORS PROFILE}

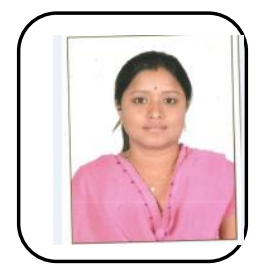

Ms. Nirupama S Graduated in B.E.(ISE) from VTU Belagavi in 2009 and received Masters Degree in M.Tech.(CSE), from VTU Belagavi, in 2012. She is currently attached with Sreenidhi Institute Of Science \& Technology in Hyderabad. Presently she is working as a Assistant Professor in IT department. Her areas of interest include Data Mining, Information Security, WSN and computer networks.

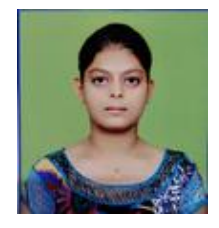

Ms. Pamireddy Sindhu, is a B.Tech (IT) student in Sreenidhi Institute of Science and Technology, Yamnampet, Ghatkesar, Hyderabad. She is studying in the Department of Information Technology. Her Area of Interest subjects are Artificial Intelligence, Robotics, Machine Learning, IOT applications and Network Security.

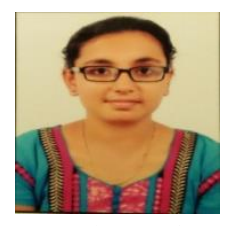

Ms. Nethi Divya Sri, is a B.Tech (IT) student in Sreenidhi Institute of Science and Technology, Yamnampet, Ghatkesar, Hyderabad. She is studying in the Department of Information Technology. Her Area of Interest subjects are Artificial Intelligence, Network Security Robotics, Machine Learning, IOT applications and

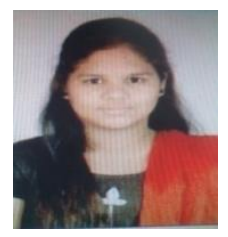

Ms. P.Lakshmi Durga Bhavani, is a B.Tech (IT) student in Sreenidhi Institute of Science and Technology, Yamnampet, Ghatkesar, Hyderabad. She is studying in the Department of Information Technology. Her Area of Interest subjects are Artificial Intelligence, Robotics, Machine Learning, IOT applications and Network Security. 